\title{
Influence of the SOD2 A16V gene polymorphism on alterations of redox markers and erythrocyte membrane fatty acid profiles in patients with multiple chemical sensitivity
}

\author{
ATTILIO CANNATA $^{1 *}$, CHIARA DE LUCA ${ }^{2 *}$, GIULIA ANDOLINA ${ }^{1}$, DANIELA CACCAMO $^{1}$, MONICA CURRÒ ${ }^{1}$, \\ NADIA FERLAZZO ${ }^{1}$, RICCARDO IENTILE ${ }^{1}$, ANGELA ALIBRANDI ${ }^{3}$ and LIUDMILA KORKINA ${ }^{4}$ \\ ${ }^{1}$ Department of Biomedical Sciences, Dental Sciences and Morpho-functional Imaging, \\ University of Messina, Polyclinic Hospital University 'G. Martino', I-98125 Messina, Italy; \\ ${ }^{2}$ R\&D Regulatory Affairs Department, Medena AG, CH-8910 Affoltern-am-Albis (ZH), Switzerland; \\ ${ }^{3}$ Department of Economics, Section of Statistical and Mathematical Sciences, University of Messina, I-98124 Messina, Italy; \\ ${ }^{4}$ Centre of Innovative Biotechnological Investigations Nanolab (CIBI-NANOLAB), 119571 Moscow, Russian Federation
}

Received June 17, 2021; Accepted September 15, 2021

DOI: 10.3892/br.2021.1477

\begin{abstract}
Chronically increased oxidative stress has been reported in patients with multiple chemical sensitivity (MCS). Recently, a single nucleotide polymorphism of the gene coding for mitochondrial superoxide dismutase (SOD2), namely the missense substitution $\mathrm{A} 16 \mathrm{~V}(\mathrm{C} 47>\mathrm{T})$ resulting in alteration of SOD2 enzyme activity, has been reported to be associated with MCS. However, the influence of SOD2 A16V genetic background on redox status of patients with MCS has not yet been investigated. Here, the results of a retrospective analysis aimed to evaluate the role of the SOD2 A16V polymorphism in the alterations of antioxidant defense markers as well as fatty acid (FA) composition of erythrocyte membranes in 67 patients with MCS matched with 55 healthy controls is reported. The mutated SOD2 V16 variant was observed more frequently in the MCS group compared with the control group, and this difference was statistically significant. The most common genotype in both groups was the heterozygous SOD2 AV16 variant, whereas the mutated SOD2 VV16 variant was more frequently observed in the MCS group, although the difference was not significant. The MCS cohort showed significantly depleted levels of plasma total antioxidant
\end{abstract}

Correspondence to: Professor Daniela Caccamo, Department of Biomedical Sciences, Dental Sciences and Morpho-functional Imaging, University of Messina, Polyclinic Hospital University 'G. Martino', Via C. Valeria 1, I-98125 Messina, Italy

E-mail: daniela.caccamo@unime.it

*Contributed equally

Key words: multiple chemical sensitivity, SOD2 A16V single nucleotide polymorphism, antioxidant defenses, plasma antioxidant activity, ubiquinol, reduced glutathione, glutathione peroxidase, polyunsaturated fatty acids, saturated fatty acids, omega 6 , omega 3 activity, ubiquinol, erythrocyte reduced glutathione and membrane polyunsaturated FA levels, coupled with significant increases in glutathione peroxidase activity, likely accounting for sustained detoxification from lipoperoxides. Notably, the highest levels of oxidative stress were found in patients with MCS bearing the genotype SOD2 AA16, whereas intermediate levels were found in patients bearing the heterozygous AV16 genotype. Healthy subjects bearing the SOD2 AA16 genotype also showed increased oxidative stress compared with carriers of other SOD2 genotypes. Despite the need for further confirmations in larger cohorts, due to MCS population genetic heterogeneity, these preliminary findings suggest that SOD2 defective activity makes certain patients with MCS more susceptible to developing oxidative stress following a chronic daily exposure to pro-oxidant insults.

\section{Introduction}

There has been increasing attention focused on multiple chemical sensitivity (MCS) in the last decade, a multi-system chronic disorder that is currently included in the broader definition of sensitivity-related illnesses (SRI), and also central sensitization syndromes, together with fibromyalgia (FM), chronic fatigue syndrome (CFS) and electromagnetic hypersensitivity (EHS) $(1,2)$.

MCS develops as a result of loss of tolerance to chronic exposure to various environmental contaminants (organophosphates, solvents, heavy metals) at concentrations below the threshold limit values considered toxic for the general population (3). The wide variety of multi-organ symptoms includes chronic muscular fatigue, bronchitis and asthma, as well as effects on eye-nose-throat, gastrointestinal, cardiac, psychosomatic, neurological, memory and mood disorders, post-traumatic distress and autoimmunity. The presence of these symptoms varies extensively from one individual to another, based on individual sensitivity (4).

MCS has an estimated prevalence of $0.5-6.5 \%$ in patients that received a medical diagnosis, whilst self-diagnosed 
patients show a prevalence of 9.0-11.2\% in the general population (1). The main challenges hindering a correct clinical framework of MCS are: The absence of a specific and defined pathogenic mechanism or mechanisms; a range of potential triggers; an absence of dose-dependent responses to triggers; and the presence of common comorbidity features with known autoimmune diseases, such as systemic lupus erythematosus, rheumatoid arthritis or vitiligo $(5,6)$.

Due to difficulties in finding unique and differential diagnostic markers, defining MCS has been approached with genetic, metabolic, immunological, etiological, symptomatic, therapeutic and epidemiological tools (7). To date, the importance of oxidative stress as an etiopathogenetic mechanism of this condition is widely recognized, in addition to the role of redox status alterations in the development of chronic mild systemic inflammation (5-7). In previous studies it was demonstrated in the clinical setting how oxidative status and inflammatory markers were elevated in patients with MCS compared with healthy subjects (8-12). Moreover, almost all patients with MCS are characterized by a genetic background, potentially predisposing them to the development of oxidative/nitrosative stress, given the presence of several single nucleotide polymorphisms (SNPs) in genes coding for enzymes involved in phase I and II detoxification reactions, as well as antioxidant enzymes (9-11,13-17). In particular, the rs4880 SNP (C47>T) of the superoxide dismutase 2 (SOD2) gene has been reported as one of the genetic determinants of MCS risk (15). The $\mathrm{C}$ to $\mathrm{T}$ nucleotide change leads to an alanine to valine amino acid change at codon 16 (Ala16Val, A16V), leading to a reduction of SOD2 levels in the mitochondria and thus reduced superoxide removal, resulting in superoxide accumulation and mitochondrial damage (18). Notably, an impairment in liver function after exposure to bisphenol A has been reported in carriers of the SOD2 variant (19).

Despite the reported association of the SOD2 A16V polymorphism with MCS, the role of SOD2 A16V genetic background in the modifications of the redox status in patients with MCS has not yet been investigated.

Starting from biochemical features of patients with MCS characterized in our previous study (8), the influence of the SOD2 A16V polymorphism on the alterations of selected biomarkers of systemic oxidative damage in patients with MCS compared with healthy subjects were evaluated in the present study.

\section{Materials and methods}

Study cohort. The cases examined in this study consisted of 67 Italian patients with MCS (44 female/23 male; median age, 48 years; inter-quartile range, 12) randomly selected amongst patients that had been enrolled by medical staff at Department of Medical Pathophysiology, University of Rome 'La Sapienza' - Polyclinic Hospital 'Umberto I' (Rome, Italy), at Istituto Dermopatico dell'Immacolata (IDI-IRCCS, study protocol n.121/CE/2008) (Rome, Italy), and at Istituto of Ricerca Medica Ambientale (IRMA, Acireale, Italy) between 2009 and 2014.

Patients had been selected on the basis of diagnosis for MCS, established according to Cullen's criteria (20) and the Quick Environment Exposure Sensitivity Inventory (QEESI) score (21), adapted for local application. QEESI is a reference
Table I. Genetic background of the patients with MCS and healthy controls at the SOD2 locus.

\begin{tabular}{lccc}
\hline $\begin{array}{l}\text { SOD2 genotype } \\
\text { (C47>T, A16V) }\end{array}$ & CTR (n) & MCS (n) & P-value \\
\hline CC47 (AA16) & $24 \%(13)$ & $10 \%(7)$ & 0.0836 \\
CT47 (AV16) & $47 \%(26)$ & $51 \%(34)$ & 0.7197 \\
TT (VV16) & $29 \%(16)$ & $39 \%(26)$ & 0.3386 \\
C allele frequency & 0.472 & 0.358 & \\
T allele frequency & 0.527 & 0.641 & \\
\hline
\end{tabular}

SOD2, superoxide dismutase 2; CTR, controls ( $n=55)$; MCS, multiple chemical sensitivity patients $(n=67)$.

validated questionnaire to determine the levels of sensitization to chemical environmental triggers (airborne or other), to score the type, localization and severity of symptoms after exposure, and their life impact. MCS subjects are stratified based on a QEESI score $>20$.

Controls examined in this study were 55 Italian healthy subjects (44 female/11 male, median age, 53 years old; inter-quartile range, 12) randomly selected amongst volunteers that had been recruited, between 2011 and 2017, at IDI-IRCCS (Rome, Italy) (protocol approval no. 52/CE/2010) and Polyclinic Hospital University 'G. Martino' (Messina, Italy) (protocol approvals no. 37/17 and 51/17), according to the following established criteria: i) Absence of any clinically diagnosed disease, in particular allergic or immunologic disturbances; ii) no drug or nutraceutical supplement use in at least the 6 weeks prior to blood sampling; and iii) whole blood total production of reactive oxygen species/reactive nitrogen species (ROS/RNS) below $650 \mathrm{cps} / \mu 1$, as determined by luminol-dependent chemiluminescent response to phorbol-myristate-acetate (PMA) (22).

Non-smokers accounted for $76.2 \%$ of individuals in the MCS group and $90.9 \%$ of the control group. Alcohol or other drug users were absent in both groups of participants. None of the enrolled subject had taken any antioxidant supplementation in the 3 months preceding the recruitment.

All subjects enrolled for the study provided written informed consent to participate in the study and to blood sampling as well as anamnestic data collection, as specified in the Declaration of Helsinki (23).

Genotyping. Genomic DNA was isolated from frozen $\left(-80^{\circ} \mathrm{C}\right)$ blood samples using the Puregene-DNA purification system (GENTRA, Qiagen GmbH), according to manufacturer's protocol. The DNA was quantified by spectrophotometric measurement at $260 \mathrm{~nm}$ using a Biophotometer (Eppendorf). DNA quality was considered acceptable for samples with a $260 / 280$ ratio $\geq 1.6$. DNA integrity and the presence of contaminant RNA were assessed by electrophoresis on a $0.8 \%$ agarose gel, and subsequent UV detection of DNA bands using a gel photodocumentation system (Vilber Lourmat).

Genotyping of patients with MCS and controls for SOD2 A16V (C47> T, rs4880) SNP were performed using quantitative PCR-based allelic discrimination using a pre-designed 
Table II. Variability of non-enzymatic antioxidant defense biomarkers in MCS patients and healthy controls having different genetic backgrounds.

\begin{tabular}{|c|c|c|c|c|c|c|}
\hline \multirow[b]{2}{*}{ Genotype SOD2A16V } & \multicolumn{2}{|c|}{$\begin{array}{l}\text { Erythrocyte content of } \\
\text { reduced glutathione, } \mathrm{mg} / \mathrm{l}\end{array}$} & \multicolumn{2}{|c|}{ Plasma ubiquinol, $\mu \mathrm{g} / \mathrm{l}$} & \multicolumn{2}{|c|}{ Antioxidant activity, $\mu \mathrm{mol} / 1$} \\
\hline & CTR & MCS & CTR & MCS & CTR & MCS \\
\hline AA16 & $347.0 \pm 45.5$ & $307.1 \pm 5.6$ & $578.0 \pm 95.1$ & $575.8 \pm 45.6$ & $0.66 \pm 0.30$ & $0.44 \pm 0.2^{\mathrm{a}}$ \\
\hline AV16 & $342.5 \pm 61.2$ & $331.4 \pm 40.1$ & $633.6 \pm 75.8$ & $599.2 \pm 33.0$ & $0.60 \pm 0.24$ & $0.48 \pm 0.2^{\mathrm{a}}$ \\
\hline VV16 & $342.9 \pm 60.5$ & $310.7 \pm 31.4$ & $655.7 \pm 71.8$ & $565.7 \pm 76.4^{\mathrm{b}}$ & $0.59 \pm 0.21$ & $0.47 \pm 1.6^{\mathrm{a}}$ \\
\hline
\end{tabular}

CTR, control; MCS, multiple chemical sensitivity; SOD2, superoxide dismutase 2. A Mann-Whitney U test for independent samples was employed to perform comparisons between cases and controls. ${ }^{\mathrm{a}} \mathrm{P}<0.05,{ }^{\mathrm{b}} \mathrm{P}<0.01$ vs. respective CTR.

TaqMan SNP genotyping assay (ID: C_8709053_10), available from Applied Biosystems (Thermo Fisher Scientific, Inc.) according to the manufacturer's protocol.

Genotyping was performed on a 7900HT Fast Real-Time PCR system (Applied Biosystems; Thermo Fisher Scientific, Inc.), using thermal cycling conditions as previously described (24).

Analysis of redox markers. The whole blood luminol-dependent chemiluminescence $(\mathrm{CL})$ response to PMA and the plasma levels of nitrites/nitrates $\left(\mathrm{NO}_{2}{ }^{-} / \mathrm{NO}_{3}{ }^{-}\right)$reacting with Griess reagent were measured as described previously by $(22,25)$.

The levels of reduced glutathione (GSH) in erythrocytes, as well as the plasma concentrations of ubiquinol (Ubi-ol), the reduced form of coenzyme Q10 $\left(\mathrm{CoQ}_{10} \mathrm{H}_{2}\right)$, were assessed by High-Performance Liquid Chromatography equipped with array photodiode and electrochemical detection, as previously described (7).Enzyme activities of superoxide dismutase (SOD), catalase (CAT), glutathione-S-transferase (GST) and glutathione peroxidase (GPx) in erythrocytes were measured spectrophotometrically, as described previously (26-29). The antioxidant activity (AOA) in plasma was determined as described (30).

Analysis of fatty acid (FA) levels in erythrocyte membranes. The pattern of FA esterified to the phospholipids of the erythrocyte membranes was analyzed by gas chromatography coupled with mass spectrometry on a crosslinked-FFAP capillary column, following purification of lipid fractions by thin layer chromatography (TLC), as described (31). Results were expressed as a percentage of the total FA content.

Statistical analysis. Continuous data were expressed as the mean \pm standard deviation, and the categorical variables as the number and percentage. The difference between cases and controls, in terms of categorical variables, was assessed using a Fisher's exact test, as well as the compliance of genotype distributions to the Hardy-Weinberg equilibrium, using an online tool (ihg.gsf.de/cgi-bin/hw/hwa1.pl). The distribution of data was assessed using a Kolmogorov-Smirnov test, and was found to exhibit skewed distribution. Thus, non-parametric tests were used for subsequent statistical analyses. A Mann-Whitney U test for independent samples was used to compare comparisons between cases and controls. Statistical analysis was performed using SPSS version 22.0 (IBM Corp.). $\mathrm{P}<0.05$ was considered to indicate a statistically significant difference.

\section{Results}

Genotype distributions for SOD2 A16V polymorphism in patients with MCS and healthy controls are shown in Table I. Genotype frequencies, both in cases and controls, were in Hardy-Weinberg equilibrium and, similarly, allele frequencies amongst cases and controls were within the $95 \%$ confidence interval.

The analysis of the SOD2 A16V $(\mathrm{C}>\mathrm{T})$ genotype distribution showed that the AV heterozygous genotype was the most represented in both populations, as compared with other SOD2 genotypes, even if it was slightly more frequent in the MCS group compared with the control group (Table I).

The AA16 genotype was observed more frequently amongst healthy subjects vs. patients, whereas the TT16 genotype was more frequently observed in patients with MCS compared with the controls. However, no statistically significant differences were found between cases and controls.

Comparative analysis of metabolic redox biomarkers and FA profiles was performed, as a whole and also related to the different SOD2 A16V genetic background of the patients with MCS and healthy controls.

MCS cases displayed a severely reduced antioxidant capacity as compared to healthy controls, as evidenced by significantly lower plasma AOA values $(0.42 \pm 0.18$ vs. $0.61 \pm 0.24 \mathrm{mmol} / \mathrm{l}$; $\mathrm{P}=0.000$ ). A significantly lower AOA level was observed in patients with MCS with either the AA or AV genotype, compared with their healthy counterparts $(\mathrm{P}<0.05$, Table II).

The concentration of Ubi-ol, was also significantly depleted in patients with MCS vs. healthy subjects $(573.9 \pm 99.40$ vs. $626.92 \pm 86.55 \mu \mathrm{g} / \mathrm{l} ; \mathrm{P}=0.007)$, along with erythrocyte content of GSH $(297.0 \pm 68.0$ vs. $343.72 \pm 53.50 \mathrm{mg} / 1 ; \mathrm{P}=0.008)$, accounting for the impaired antioxidant capacity typical in patients with this condition. Patients with MCS with the AA genotype showed the lowest levels of GSH and Ubi-ol, lower than those observed in the controls, although the differences were not statistically significant.

PMA-triggered release of ROS/RNS from granulocytes, as non-specifically determined by PMA-induced-CL 
Table III. Variability of antioxidant enzyme activities in patients with MCS and CTR with different SOD2 A16V genotypes.

\begin{tabular}{|c|c|c|c|c|c|c|c|c|}
\hline \multirow[b]{2}{*}{ Genotype SOD2 A16V } & \multicolumn{2}{|c|}{$\begin{array}{c}\text { Superoxide } \\
\text { dismutase, } \mathrm{U} / \mathrm{g} \text { prot }\end{array}$} & \multicolumn{2}{|c|}{$\begin{array}{l}\text { Catalase, } \\
\text { U/g prot }\end{array}$} & \multicolumn{2}{|c|}{$\begin{array}{c}\text { Glutathione } \\
\text { peroxidase, } \mathrm{U} / \mathrm{mg} \mathrm{Hb}\end{array}$} & \multicolumn{2}{|c|}{$\begin{array}{c}\text { Glutathione-S } \\
\text {-transferase, } \mathrm{U} / \mathrm{mg} \mathrm{Hb}\end{array}$} \\
\hline & CTR & MCS & CTR & MCS & CTR & MCS & CTR & MCS \\
\hline AA & $0.33 \pm 0.1$ & $0.27 \pm 0.3$ & $8.5 \pm 3.8$ & $6.7 \pm 0.3$ & $22.8 \pm 10$ & $25.4 \pm 2.3$ & $2.0 \pm 0.7$ & $1.6 \pm 0.6$ \\
\hline AV & $0.31 \pm 0.3$ & $0.29 \pm 0.1$ & $8.6 \pm 2.7$ & $7.8 \pm 1.5$ & $19.4 \pm 7.0$ & $27.0 \pm 4.0^{\mathrm{a}}$ & $1.9 \pm 0.8$ & $1.8 \pm 0.2$ \\
\hline VV & $0.23 \pm 0.1$ & $0.28 \pm 0.1$ & $8.4 \pm 3.9$ & $8.1 \pm 2.1$ & $23.4 \pm 7.6$ & $21.1 \pm 4.6$ & $1.9 \pm 0.5$ & $1.8 \pm 0.4$ \\
\hline
\end{tabular}

${ }^{a} \mathrm{P}<0.01$ vs. respective CTR. CTR, control; MCS, multiple chemical sensitivity. A Mann-Whitney U test for independent samples were employed to perform comparisons between cases and controls.

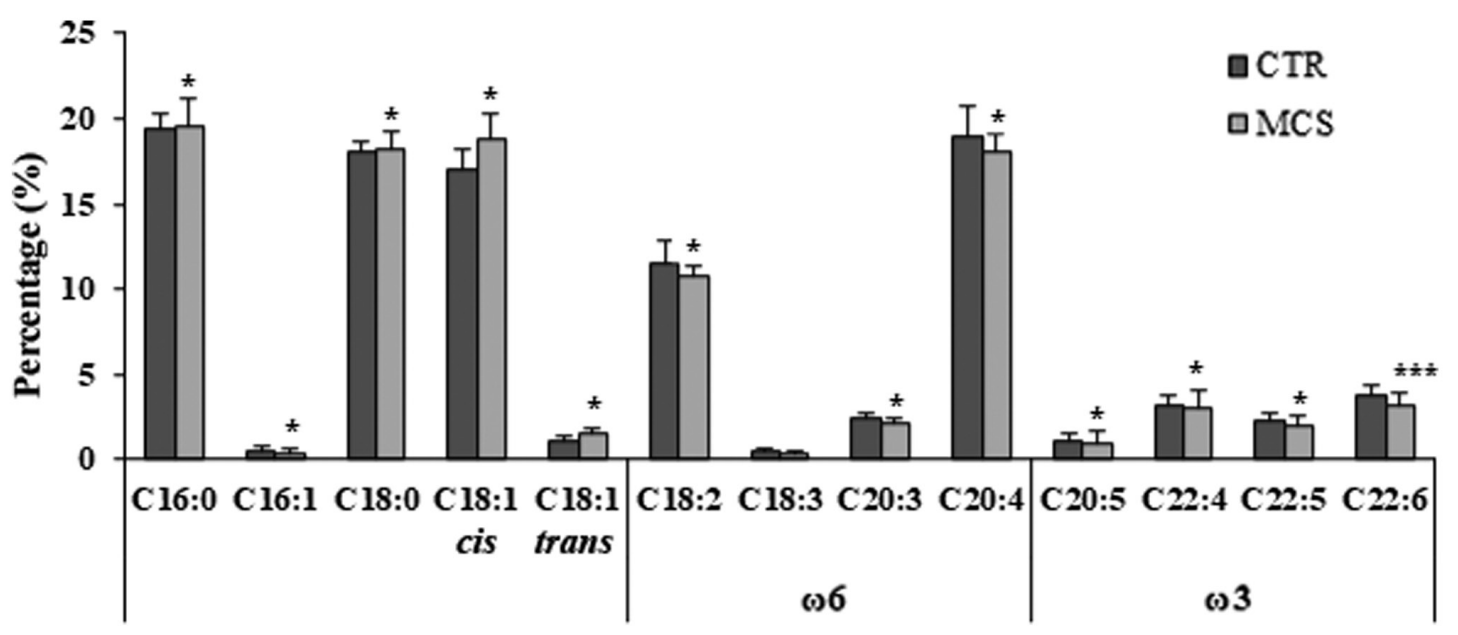

Figure 1. Fatty acids composition of erythrocyte membranes in MCS cases and healthy controls. Relative abundance (\%) of representative saturated fatty acids (C16:0, palmitic acid; C18:0, stearic acid), monounsaturated fatty acids (C16:1, palmitoleic acid; cis C18:1 oleic acid; trans $\mathrm{C} 18: 1$, elaidic acid) and polyunsatured fatty acids ( $\omega 6 \mathrm{C} 18: 2$, linoleic acid; $\omega 6 \mathrm{C} 18: 3, \gamma$-linolenic acid; $\omega 6 \mathrm{C} 20: 3$, dihomo- $\gamma$-linolenic acid; $\omega 6 \mathrm{C} 20: 4$, arachidonic acid; $\omega 3 \mathrm{C} 20: 5$, eicosapentaenoic acid; $\omega 3$ C22:4, docosatetraenoic acid (DTA); $\omega 3$ C22:5, docosapentaenoic acid (DPA); $\omega 3$ C22:6, cervonic acid. A Mann-Whitney U test for independent samples was used to make comparisons between the cases and controls. ${ }^{*} \mathrm{P}<0.05,{ }^{* * *} \mathrm{P}<0.01$ vs. respective CTR value. MCS, multiple chemical sensitivity patients ( $\mathrm{n}=67)$; CTR, controls $(\mathrm{n}=55)$.

$(416.3 \pm 160.9$ vs. $386.6 \pm 140.2 \mathrm{cps} / \mu 1, \mathrm{MCS}$ vs. control), and the $\mathrm{NO}_{2} / \mathrm{NO}_{3}$ - plasma levels $(23.4 \pm 130.9$ vs. $18.9 \pm 6.6 \mu \mathrm{mol} / 1$, MCS vs. control), an indirect measurement of the extracellular superoxide anion and nitric oxide production by circulating leukocytes and endotheliocytes, were not significantly different between the two groups.

Moreover, the erythrocyte antioxidant GPx activity was significantly higher in patients with MC compared with the controls $(25.44 \pm 8.12$ vs. $21.39 \pm 7.67 \mathrm{U} / \mathrm{mg} \mathrm{Hb} ; \mathrm{P}=0.018)$. The SOD antioxidant activity was found to be increased in MCS cases compared with controls $(0.27 \pm 0.33$ vs. $0.25 \pm 0.07 \mathrm{U} / \mathrm{g}$ prot; $\mathrm{P}=0.064)$, while CAT activity was higher in controls than in patients with $\mathrm{MCS}(8.54 \pm 3.86 \mathrm{vs}$. $7.40 \pm 5.45 \mathrm{U} / \mathrm{g}$ prot; $\mathrm{P}=0.057)$. Despite being not significant, the $\mathrm{P}$-values associated with these differences may suggest a trend towards statistical significance. GST enzyme activity was lower in patients with MCS compared with healthy controls (1.70 \pm 0.62 vs. $1.93 \pm 0.69 \mathrm{U} / \mathrm{mg} \mathrm{Hb} ; \mathrm{P}=0.101)$.

No significant differences were found between genotype subgroups in MCS cases and control subjects with regard to antioxidant enzyme activities, except for erythrocyte GPx activity, which was significantly increased in patients bearing the AV16 genotype compared with their healthy counterparts (Table III).

The FA composition of the phospholipids in the erythrocyte membrane of patients with MCS and controls, as possible markers of oxidative damage.

The comparative analysis of FA profiles showed that the percentage of saturated FA (SFA) was significantly higher in patients with MCS compared with the controls (38.31 \pm 2.74 vs. $37.5 \pm 1.02 \%$; $\mathrm{P}=0.041)$. Patients with MCS also showed a significantly higher concentration of monounsaturated fatty acids (UFA) compared with controls $(20.03 \pm 2.25$ vs. $18.63 \pm 1.35 \% ; \mathrm{P}=0.035)$. SFA was primarily represented by palmitic acid (C16:0) and stearic acid (C18:0) in patients with MCS (Fig. 1).

As a key result relevant to the measurement of oxidative damage, the percentage of the highly oxidable polyunsaturated fatty acids (PUFA) was significantly lower in patients with MCS compared with the controls $(43.3 \pm 1.6$ vs. $41.7 \pm 1.8 \%$; $\mathrm{P}=0.021)$. In particular, the levels of omega-6 PUFA, linoleic acid (C18:2 $\omega 6)$, arachidonic acid (C20:4 $\omega 6)$ and dihomo- $\gamma$-linolenic acid (C20:3 $\omega 6)$, as well as the omega-3 FA, eicosapentaenoic acid (C20:5 13 ), docosatetraenoic acid 
Table IV. Variability of erythrocyte membrane phospholipid fatty acid composition in patients with MCS and CTR with a different SOD2 genetic background.

\begin{tabular}{|c|c|c|c|c|c|c|}
\hline \multirow[b]{2}{*}{ Fatty acid } & \multicolumn{3}{|c|}{ CTR } & \multicolumn{3}{|c|}{ MCS } \\
\hline & SOD2 AA16 & SOD2 AV16 & SOD2 VV16 & SOD2 AA16 & SOD2 AV16 & SOD2 VV16 \\
\hline $\mathrm{C} 16: 0, \%$ & $19.6 \pm 0.9$ & $20.0 \pm 1.1$ & $19.9 \pm 1.1$ & $20.7 \pm 0.9^{b}$ & $19.3 \pm 0.4^{\mathrm{a}}$ & $19.1 \pm 1.1$ \\
\hline C16:1,\% & $0.5 \pm 0.3$ & $0.5 \pm 0.3$ & $0.4 \pm 0.3$ & $0.4 \pm 0.1$ & $0.3 \pm 0.1$ & $0.3 \pm 0.0$ \\
\hline $\mathrm{C} 18: 0, \%$ & $18.0 \pm 0.6$ & $18.4 \pm 0.8$ & $18.4 \pm 0.6$ & $18.6 \pm 1.2$ & $18.0 \pm 1.4$ & $17.9 \pm 0.7$ \\
\hline $\mathrm{C} 18: 1 \mathrm{cis}, \%$ & $16.9 \pm 1.5$ & $17.1 \pm 1$ & $17 \pm 1.1$ & $18.1 \pm 0.7$ & $17.5 \pm 1.1$ & $18.1 \pm 1.0^{\mathrm{a}}$ \\
\hline $\mathrm{C} 18: 1$ trans, $\%$ & $1.2 \pm 0.4$ & $1.0 \pm 0.2$ & $1.1 \pm 0.1$ & $1.3 \pm 0.4$ & $1.3 \pm 0.4^{\mathrm{b}}$ & $1.4 \pm 0.5 b$ \\
\hline $\mathrm{C} 18: 2, \%$ & $11.7 \pm 1.1$ & $11.4 \pm 1.7$ & $11.4 \pm 0.9$ & $10.4 \pm 0.9^{b}$ & $11.2 \pm 1.1$ & $11.3 \pm 0.9$ \\
\hline $\mathrm{C} 18: 3, \%$ & $0.42 \pm 0.2$ & $0.5 \pm 0.2$ & $0.51 \pm 0.7$ & $0.38 \pm 0.1$ & $0.42 \pm 0.1^{\mathrm{a}}$ & $0.44 \pm 0.7^{\mathrm{a}}$ \\
\hline $\mathrm{C} 20: 3, \%$ & $2.37 \pm 0.4$ & $2.56 \pm 0.3$ & $2.27 \pm 0.3$ & $2.3 \pm 0.3$ & $2.4 \pm 1.0$ & $2.2 \pm 0.3$ \\
\hline $\mathrm{C} 20: 4, \%$ & $18.7 \pm 1.8$ & $19.5 \pm 7.9$ & $19.1 \pm 1.4$ & $18.2 \pm 1.2$ & $18.2 \pm 1.8^{\mathrm{a}}$ & $19.0 \pm 1.2$ \\
\hline $\mathrm{C} 20: 5, \%$ & $1.28 \pm 0.6$ & $1.0 \pm 0.2$ & $0.9 \pm 0.4$ & $0.85 \pm 0.3$ & $0.9 \pm 0.4$ & $1.0 \pm 0.3$ \\
\hline $\mathrm{C} 22: 4, \%$ & $3.2 \pm 0.8$ & $3.0 \pm 0.6$ & $3.2 \pm 0.5$ & $2.9 \pm 0.4$ & $3.0 \pm 0.4$ & $3.1 \pm 0.4$ \\
\hline $\mathrm{C} 22: 5, \%$ & $2.3 \pm 0.4$ & $2.2 \pm 0.6$ & $2.2 \pm 0.5$ & $2.0 \pm 0.4$ & $2.2 \pm 0.5$ & $2.1 \pm 0.5$ \\
\hline $\mathrm{C} 22: 6, \%$ & $3.8 \pm 0.8$ & $3.9 \pm 0.7$ & $3.7 \pm 0.8$ & $3.2 \pm 1.9$ & $3.6 \pm 0.5$ & $3.8 \pm 0.5$ \\
\hline SFA, \% & $38.2 \pm 1.4$ & $37.4 \pm 1.6$ & $37.0 \pm 1.3$ & $39.2 \pm 1.9$ & $38.4 \pm 1.7$ & $38.3 \pm 2.0$ \\
\hline UFA, \% & $18.5 \pm 1.6$ & $18.7 \pm 1.2$ & $18.5 \pm 1.3$ & $29.6 \pm 4.2$ & $19.1 \pm 0.6$ & $22.0 \pm 1.4$ \\
\hline PUFA, \% & $43.3 \pm 1.7$ & $43.6 \pm 1.8$ & $44.0 \pm 1.9$ & $40.0 \pm 1.8$ & $41.2 \pm 0.9$ & $41.9 \pm 0.7$ \\
\hline$\omega 6 / \omega 3$ & $3.1 \pm 0.5$ & $3.2 \pm 0.4$ & $3.3 \pm 0.5$ & $3.7 \pm 0.4$ & $3.4 \pm 0.5$ & $3.6 \pm 0.7$ \\
\hline
\end{tabular}

${ }^{\mathrm{a}} \mathrm{P}<0.05,{ }^{\mathrm{b}} \mathrm{P}<0.01$ vs. respective CTR. CTR, control; MCS, multiple chemical sensitivity; SFA, saturated fatty acids; UFA, unsaturated fatty acids; PUFA, polyunsaturated fatty acids. SFA: C16:0, palmitic acid; C18:0, stearic acid. monounsaturated fatty acids: C16:1, palmitoleic acid; cis C18:1 oleic acid; trans C18:1, elaidic acid. Polyunsatured $\omega 6$ fatty acids: C18:2, linoleic acid; $\omega 6$ C18:3, $\gamma$-linolenic acid; $\omega 6$ C20:3, dihomo- $\gamma$-linolenic acid; $\omega 6$ C20:4, arachidonic acid. Polyunsatutared $\omega 3$ fatty acids: C20:5, eicosapentaenoic acid; $\omega 3$ C22:4, docosatetraenoic acid; $\omega 3 \mathrm{C} 22: 5$, docosapentaenoic acid; $\omega 3 \mathrm{C} 22: 6$, cervonic acid. A Mann-Whitney U test for independent samples were employed to perform comparisons between cases and controls.

(DTA C22:4 $\omega 3$ ), docosapentaenoic acid (C22:5 $\omega 3)$ and docosahexaenoic acid (C22:6 $\omega 3)$, were significantly reduced in the MCS group (Fig. 1). Finally, the $\omega 6 / \omega 3$ ratio was significantly higher in patients with MCS compared with the controls (3.68 \pm 0.95 vs. $3.36 \pm 0.60 \% ; \mathrm{P}=0.038)$.

The MCS group presented with no overall functionally relevant differences in the FA profiles between patient and control groups (Table IV). Minor significant differences were observed in individuals possessing a AA genotype, with a significantly increased percentage of the amount of palmitic acid (C16:0) and, at to a minor extent, stearic acid (C18:0) (Table IV), and significantly lower amounts of linoleic acid (18:2) (Table IV), the progenitor of the $\omega-6$ series, in comparison with healthy subjects. Instead, the monounsaturated elaidic acid (trans C18:1) was significantly increased in MCS cases possessing either the VV or the AV genotype compared with controls (Table IV), while the $\omega-6 \gamma$-linolenic acid (C18:3) levels were significantly lower in the same patients (Table IV). Moreover, oleic acid (cis C18:1) levels were significantly higher in the patients with MCS with the VV genotype compared with the controls (Table IV). The amount of $\omega 6$ arachidonic acid was reduced in all patients, and significant differences were found only in those possessing an AV genotype compared with controls.

All FAs of the $\omega-3$ series were found to be depleted in patients with MCS compared with controls, and the lowest levels were found in patients with SOD2 AA16 genotype (Table IV). However, no significant differences were observed between cases and controls.

SFA and UFA were increased in patients with MCS compared with the controls, and the highest percentage amounts were found in subjects with the AA genotype, whereas in the same individuals, the lowest levels of PUFA were observed, that were generally reduced in comparison with healthy subjects. Notably, the ratio $\omega 6 / \omega 3$ was found to be higher in MCS cases compared with the healthy controls, and the highest values were observed in patients with the SOD2 AA16 genotype (Table IV). However, no significant differences were observed between the two groups, and only a trend to statistical significance was observed for differences in SFA amounts in patients with MCS with either the AV genotype $(\mathrm{P}=0.05)$ or the VV genotype $(\mathrm{P}=0.07)$.

\section{Discussion}

In the last two decades several studies have provided evidence for a correlation between MCS and chemical defense system alterations, occurring in the presence of gene polymorphisms of detoxification phase I (CYPs) and phase II enzymes (GST, NAT and UGT, amongst others), as well as antioxidant enzymes SOD2 and GPX (5,9-17). Previous study by De Luca et al (8) on a large group of patients with MCS 
highlighted the relevance of oxidative stress in this syndrome. In particular, a reduction of CAT and GST enzyme activities, associated with glutathione reduction, increased GPx activity, PUFA-depleted profiles of erythrocyte membranes, and specifically altered pro-inflammatory plasma cytokine patterns have been shown in a MCS cohort as a whole $(8,9)$. Moreover, in our previous study, it was reported that there was a significantly higher frequency of polymorphisms in genes coding for CYP enzymes in patients with MCS compared with controls. These findings indicated that these genetic variants may increase the individuals' susceptibility to develop MCS, and oxidative stress conditions typically associated with this complex syndrome (14). Notably, Cui et al (15) also reported that the missense polymorphism A16V in gene SOD2, coding for the antioxidant mitochondrial superoxide dismutase, is associated with MCS development. In silico and in vitro experiments showed that this polymorphism can significantly affect enzyme activity, reducing antioxidant defenses (15). However, the relationship between SOD2 genetic background and redox metabolism features in patients with MCS have not been investigated thus far, to the best of our knowledge.

In the present study, the role of SOD2 A16V polymorphism in the modifications was examined in the previously established panel of redox biomarkers assessed in a representative group of 67 patients with MCS as compared with an age-matched group of 55 healthy controls. The goal was to search for possible correlations between the inter-individual variations existing in the blood metabolic parameters and the genetic background.

Taking into consideration the MCS group as a whole, there was a reduced capacity of the antioxidant system of these individuals compared with the healthy counterparts, as evidenced by the decreased values of plasma AOA and Ubi-ol, as well as erythrocyte GSH. GSH represents the most powerful antioxidant agent, capable of preventing ROS-induced damage to important cellular components, and is also largely used for cell and body detoxification from xenobiotics (5). Ubi-ol is a potent endogenous antioxidant, and its depletion is viewed as a reliable biomarker of systemic oxidative stress (7). It acts as a reducing agent in the mitochondria and in lipid membranes by either directly scavenging free radicals or in conjunction with $\alpha$-tocopherol (5). These results confirmed the findings of Miyamae et al (32) with fibromyalgic patients, that showed a drastic reduction of Ubi-ol levels compared with healthy controls, suggesting an increased formation of ROS in the circulating blood in patients with juvenile FM.

The measurement of antioxidant enzymes SOD, CAT and GPx in erythrocytes allows assessment, in a non-invasive manner, of the circulating antioxidant defense in humans. Cell damage by ROS is initiated by the production of the superoxide radicals, which is closely associated with the metabolism of molecular oxygen in mitochondria and in cellular membranes. Thus, the first-line antioxidant defense system against oxidative damage is represented by SOD, the cytosolic $\mathrm{Cu}-\mathrm{Zn}$ SOD (SOD1, dimeric), the mitochondrial Mn-SOD (SOD2), and the extracellular $\mathrm{Cu}-\mathrm{Zn}$ SOD (SOD3, tetrameric), which convert superoxide anion to hydrogen peroxide. Subsequently, hydrogen peroxide is scavenged by CAT and GPx (33). Although it was not significant, there was a trend-to-reduction of CAT and GST activities, and a trend-to-increase in SOD enzyme activity in patients with MCS, coupled with an overall significant glutathione depletion. Both CAT and GST are stress proteins that are upregulated under pathological conditions, such as that represented by chemical stress. However, chemical stress can also induce cytotoxicity in blood cells producing CAT, that in turn may result in a reduction in CAT activity and accumulation of excessive amounts of hydrogen peroxide. Hydrogen peroxide excess initiates a chain-reaction of lipid peroxidation, the major feature of which is the decomposition of PUFA to aldehydes, with an overall reduction of PUFA content in erythrocyte membranes. Under these conditions, GPx likely becomes the major second-line antioxidant enzyme to neutralize hydrogen peroxide through the oxidation of GSH to oxidized glutathione, and counteract the accelerated production of lipid hydroperoxides. These observations highlight a general trend of increased oxidative stress in patients with MCS. As a likely consequence, patients with MCS presented with markedly increased GPx activity and GSH depletion (5). Consistently, a significant increase in GPx activity has been previously confirmed in the muscles of patients with CFS, sharing several symptomatic features with patients with MCS (34). This possible adaptive response of GPx to excess hydroperoxides is in line with previous findings of our group in psoriasis, a pathological condition characterized by chronic inflammation and immune system activation (35). Notably, the genetic background at the GPx locus does not seem to be altered in patients with MCS as compared with the healthy population, at least as concerns the $\mathrm{rs} 1800668(\mathrm{C} / \mathrm{T})$ variant within the promoter of GPx1 gene, which is able to affect enzyme activity (11).

The increased lipid peroxidation observed in the MCS group was also indirectly confirmed by the observed alterations in the FA profiles of red blood cell membranes. The contents of all PUFAs and of selected PUFAs relevant to inflammatory/anti-inflammatory processes, primarily arachidonic (AA; C20:4, $\omega 6$ ) were much lower-than-normal in the MCS cohort, thus confirming the occurrence of a sustained lipoperoxidation in the erythrocyte membranes of patients with MCS.

A key chemical feature of lipid peroxidation is the ability to decompose PUFA to form a broad array of end-products, namely aldehydes, such as such as malondialdehyde and 4-hydroxy-2-nonenal (4-HNE), a stable electrophile formed during the lipid peroxidation of $\omega-6$-PUFA, namely linoleic and arachidonic acids, which readily react with proteins and DNA to affect enzyme gene expression, enzymatic activity, as well as the formation of autoantigens (36).

A similar pattern of increased GPx activity and depleted erythrocyte membrane PUFA, with increased levels of end-products of lipoperoxidation, was shown in patients affected with psoriasis, a chronic inflammatory immune-mediated pathology, which shares with MCS a severe imbalance of the systemic redox status coupled with a marked dysregulation of plasmatic inflammatory cytokines (37).

The condition of impaired redox status and reduced antioxidant capacity, seen at a general level in the MCS group, was also found considering the different genotypic structures of SOD2 A16V polymorphism in both cohorts under study. Indeed, all four major markers found as depleted in the overall MCS group, AOA, GSH, Ubi-ol and PUFA levels, along with 
increased GPx activity, showed the same trend in the MCS subgroup with the SOD2 AA genotype. This subgroup showed the largest differences between patients with MCS and controls, and also the most differing prevalence between patients and controls. Conversely, even healthy controls bearing this genotype showed oxidative stress levels higher than those found in other control subgroups with different genotypes. Notably, the lowest levels of oxidative stress in both study cohorts were found in individuals bearing the SOD2 VV16 genotype.

These findings suggest that the SOD2 AA16 wild-type genotype represents a genetic risk factor for increased susceptibility to oxidative stress, while the mutated homozygous SOD2 VV16 displays a protective effect. The Ala16-wild-type variant of SOD2 gene allows a more efficient importation of $\mathrm{Mn}-\mathrm{SOD}$ into the mitochondria, resulting in turn in the generation of a more active enzyme compared with the Val16-variant, that is related to the induction of oxidative stress. Likely, this is the reason why the SOD2 Ala16 variant has a much lower prevalence than the Val16 one in all ethnic groups worldwide (18). Notably, the heterozygous AV16 genotype seems to represent the better conditions for optimal enzyme activity of mitochondrial Mn-SOD, given the highest frequency observed in our study cohorts, that is in line with findings from several studies carried out in Italian and Caucasian populations (23,37-41). Most importantly, these same studies, and several others not cited here, suggested that the SOD2 AA16V (rs4880) polymorphism may have an impact on acute and chronic oxidative-related damage, and increase the susceptibility to various oxidative stress-related pathological conditions, such as pregnancy complications, cardio- and cerebrovascular disorders, cancer, glaucoma, diabetes $(37,38,40-43)$, and even accelerates telomere shortening with age (39).

These findings are in line with the report of Cui et al (15) suggesting that SOD2 AA16 genotype increase the risk for MCS, even if no correlation with biochemical features were assessed. Notably, it has been reported that this genotype increases the susceptibility to oxidative stress-related cyto-genotoxicity induced by pyridostigmine bromide, that has been implicated as a causal factor in Gulf War syndrome, a disorder sharing several features with MCS (44). Moreover, cytotoxic effects, at all times of exposure to static magnetic field (SMF), have been observed in peripheral blood mononuclear cells isolated from individuals bearing the AA16 genotype, while AV and VV cells presented mortality only after longer times of exposure to SMF. These results suggest a toxico-genetic effect of SMF exposure related to an imbalance in SOD2 activity associated with the AA16 genotype (45). Interestingly, hypersensitivity to electromagnetic field, also called EHS, is a common co-morbidity of $\operatorname{MCS}(9,46)$ in which oxidative stress plays a major role (47).

In conclusion, the analysis of the metabolic markers of antioxidant defense and redox imbalance confirmed the occurrence in the MCS group of a statistically solid reduction of the main four blood metabolic markers of oxidative stress, the depletion of the low-molecular weight antioxidant Ubi-ol, of the total plasmatic antioxidant activity, of the red blood cell membrane PUFA, with possible generation of lipid hydroperoxide by-products leading to an increase of GPx activity. This specific pattern of metabolic alterations was more striking in MCS carriers of the SOD2AA genotype than either in other patients or controls.
The results of the present study provide additional evidence that functional and/or genetic defects of endogenous enzymes detoxifying $\mathrm{H}_{2} \mathrm{O}_{2}$, lipid peroxides, or stable toxic products of lipid peroxidation may cause chronic oxidative stress with increased pro-inflammatory cytokine release and consequent metabolic alterations, characteristic for the patients with SRI, as originally hypothesized by our team (5). The observed correlation of these selected metabolic alterations with SOD2 A16V polymorphism may contribute to an improved understanding of the abnormal susceptibility to low-level xenobiotic stimuli in these peculiar clinical settings.

These results await confirmation by larger studies where the patients with MSC shall be necessarily further stratified based on the severity of their clinical manifestations and on their specific patterns of co-morbidities, thus taking into account the large amount of heterogeneity of MCS pathogenesis and the persisting lack of consensus on the diagnostic protocols (48). Additional confirmatory studies will possibly contribute to finally validate the clinical relevance of the described targeted panel of gene polymorphisms and of biomarkers of the systemic redox status impairment, as a feasible laboratory approach for MCS management, for an evidence-based process of diagnosis, prognosis and treatment follow-up of this multi organ syndrome.

\section{Acknowledgements}

Dr Maria Grazia Bruccheri for helping in the recruitment of patients with MCS at IRMA (Acireale, CT).

\section{Funding}

No funding was received.

\section{Availability of data and materials}

The datasets used and/or analyzed during the present study are available from the corresponding author on reasonable request.

\section{Authors' contributions}

LK and DC conceived the study. LK, MC, NF and RI performed patient/volunteer recruitment. CDL, AC and GA performed the investigations. AA analyzed the data. AC and GA prepared tables and figures. AC and CDL wrote the manuscript. DC and LK reviewed and edited the manuscript. All authors read and approved the final manuscript. AC, CDL, GA, DC, MC, NF, RI, AA and LK confirm the authenticity of all the raw data.

\section{Ethics approval and consent to participate}

The recruitment of patients and controls was approved by the Ethics Committee of IDI-IRCCS (approval no. 121/CE/2008 and 52/CE/2010) (Rome, Italy) and the Ethics Committee of Polyclinic Hospital University 'G. Martino' (approval nos. 37/17 and 51/17) (Messina, Italy). All subjects enrolled for the study provided written informed consent to participate in the study and to blood sampling as well as anamnestic data collection, as specified in the Declaration of Helsinki. 


\section{Patient consent for publication}

Not applicable.

\section{Competing interests}

The authors declare that they have no competing interests.

\section{References}

1. Genuis SJ: Sensitivity-related illness: The escalating pandemic of allergy, food intolerance and chemical sensitivity. Sci Total Environ 408: 6047-6061, 2010.

2. Yunus MB: Central sensitivity syndromes: A new paradigm and group nosology for fibromyalgia and overlapping conditions and the related issue of disease versus illness. Semin Arthritis Rheum 37: 339-352, 2008.

3. Miller CS: Toxicant-induced loss of tolerance - an emerging theory of disease? Environ Health Perspect 105 (Suppl 2): 445-453, 1997.

4. Lacour M, Zunder T, Schmidtke K, Vaith $P$ and Scheidt $C$ : Multiple chemical sensitivity syndrome (MCS) - suggestions for an extension of the U.S. MCS-case definition. Int J Hyg Environ Health 208: 141-151, 2005.

5. Korkina L, Scordo MG, Deeva I, Cesareo E and De Luca C: The chemical defensive system in the pathobiology of idiopathic environment-associated diseases. Curr Drug Metab 10: 914-931, 2009.

6. Pall ML: Multiple chemical sensitivity: Toxicological questions and mechanisms. In: General, Applied and Systems Toxicology. 3rd edition. John Wiley \& Sons (eds). John Wiley \& Sons, Ltd. Hoboken, NJ, pp2303-2352, 2009.

7. De Luca C, Raskovic D, Pacifico V, Thai JCS and Korkina L: The search for reliable biomarkers of disease in multiple chemical sensitivity and other environmental intolerances. Int J Environ Res Public Health 8: 2770-2797, 2011.

8. De Luca C, Scordo MG, Cesareo E, Pastore S, Mariani S, Maiani G, Stancato A, Loreti B, Valacchi G, Lubrano C, et al: Biological definition of multiple chemical sensitivity from redox state and cytokine profiling and not from polymorphisms of xenobiotic-metabolizing enzymes. Toxicol Appl Pharmacol 248: 285-292, 2010

9. De Luca C, Thai JC, Raskovic D, Cesareo E, Caccamo D, Trukhanov A and Korkina L: Metabolic and genetic screening of electromagnetic hypersensitive subjects as a feasible tool for diagnostics and intervention. Mediators Inflamm 2014: 924184, 2014.

10. De Luca C, Gugliandolo A, Calabrò C, Currò M, Ientile R, Raskovic D, Korkina L and Caccamo D: Role of polymorphisms of inducible nitric oxide synthase and endothelial nitric oxide synthase in idiopathic environmental intolerances. Mediators Inflamm 2015: 245308, 2015.

11. Gugliandolo A, Gangemi C, Calabrò C, Vecchio M, Di Mauro D, Renis M, Ientile R, Currò M and Caccamo D: Assessment of glutathione peroxidase-1 polymorphisms, oxidative stress and DNA damage in sensitivity-related illnesses. Life Sci 145: 27-33, 2016.

12. Cannata A, De Luca C, Korkina LG, Ferlazzo N, Ientile R, Currò M, Andolina G and Caccamo D: The SNP rs2298383 reduces ADORA2A gene transcription and positively associates with cytokine production by peripheral blood mononuclear cells in patients with Multiple Chemical Sensitivity. Int J Mol Sci 21: 1858,2020

13. Schnakenberg E, Fabig KR, Stanulla M, Strobl N, Lustig M, Fabig N and Schloot W: A cross-sectional study of self-reported chemical-related sensitivity is associated with gene variants of drug-metabolizing enzymes. Environ Health 6: 6, 2007.

14. Caccamo D, Cesareo E, Mariani S, Raskovic D, Ientile R, Currò M, Korkina L and De Luca C: Xenobiotic sensorand metabolism-related gene variants in environmental sensitivity-related illnesses: A survey on the Italian population. Oxid Med Cell Longev 2013: 831969, 2013.

15. Cui X, Lu X, Hiura M, Oda M, Miyazaki W and Katoh T: Evaluation of genetic polymorphisms in patients with multiple chemical sensitivity. PLoS One 8: e73708, 2013.

16. Micarelli A, Cormano A, Caccamo D and Alessandrini M: Olfactory-Related Quality of Life in Multiple Chemical Sensitivity: A Genetic-Acquired Factors Model. Int J Mol Sci 21: 156, 2019.
17. D'Attis S, Massari S, Mazzei F, Maio D, Vergallo I, Mauro S, Minelli M and Bozzetti MP: Assessment of CYP2C9, CYP2C19, and CYP2D6 Polymorphisms in Allergic Patients with Chemical Sensitivity. Int Arch Allergy Immunol 179: 173-186, 2019.

18. Bastaki M, Huen K, Manzanillo P, Chande N, Chen C, Balmes JR, Tager IB and Holland N: Genotype-activity relationship for Mn-superoxide dismutase, glutathione peroxidase 1 and catalase in humans. Pharmacogenet Genomics 16: 279-286, 2006.

19. Kim JH, Lee MR and Hong YC: Modification of the association of bisphenol A with abnormal liver function by polymorphisms of oxidative stress-related genes. Environ Res 147: 324-330, 2016.

20. Cullen MR: The worker with multiple chemical sensitivities: An overview. Occup Med 2: 655-661, 1987.

21. Hojo S, Kumano H, Yoshino H, Kakuta K and Ishikawa S: Application of Quick Environment Exposure Sensitivity Inventory (QEESI) for Japanese population: Study of reliability and validity of the questionnaire. Toxicol Ind Health 19: 41-49, 2003.

22. Brown GE, Silver GM, Reiff J, Allen RC and Fink MP: Polymorphonuclear neutrophil chemiluminescence in whole blood from blunt trauma patients with multiple injuries. J Trauma 46: 297-305, 1999.

23. World Medical Association: World Medical Association Declaration of Helsinki: Ethical principles for medical research involving human subjects. JAMA 310: 2191-2194, 2013.

24. Vecchio M, Currò M, Trimarchi F, Naccari S, Caccamo D, Ientile R, Barreca D and Di Mauro D: The oxidative stress response in elite water polo players: Effects of genetic background. BioMed Res Int 2017: 7019694, 2017.

25. Giovannoni G, Land JM, Keir G, Thompson EJ and Heales SJ: Adaptation of the nitrate reductase and Griess reaction methods for the measurement of serum nitrate plus nitrite levels. Ann Clin Biochem 34: 193-198, 1997.

26. Sun Y, Oberley LW and Li Y: A simple method for clinical assay of superoxide dismutase. Clin Chem 34: 497-500, 1988.

27. Aebi H: Catalase in vitro. Methods Enzymol 105: 121-126, 1984.

28. Habig WH, Pabst MJ and Jakoby WB: Glutathione S-transferases. The first enzymatic step in mercapturic acid formation. J Biol Chem 249: 7130-7139, 1974.

29. Paglia DE and Valentine WN: Studies on the quantitative and qualitative characterization of erythrocyte glutathione peroxidase. J Lab Clin Med 70: 158-169, 1967.

30. Miller NJ, Rice-Evans C and Davies MJ: A new method for measuring antioxidant activity. Biochem Soc Trans 21: 95S, 1993.

31. De Luca C, Filosa A, Grandinetti M, Maggio F, Lamba M and Passi S: Blood antioxidant status and urinary levels of catecholamine metabolites in $\beta$-thalassemia. Free Radic Res 30: 453-462, 1999.

32. Miyamae T, Seki M, Naga T, Uchino S, Asazuma H, Yoshida T, Iizuka Y, Kikuchi M, Imagawa T, Natsumeda Y, et al: Increased oxidative stress and coenzyme Q10 deficiency in juvenile fibromyalgia: Amelioration of hypercholesterolemia and fatigue by ubiquinol-10 supplementation. Redox Rep 18: 12-19, 2013.

33. Matés JM, Pérez-Gómez C and Núñez de Castro I: Antioxidant enzymes and human diseases. Clin Biochem 32: 595-603, 1999.

34. Fulle S, Mecocci P, Fanó G, Vecchiet I, Vecchini A, Racciotti D, Cherubini A, Pizzigallo E, Vecchiet L, Senin U, et al: Specific oxidative alterations in vastus lateralis muscle of patients with the diagnosis of chronic fatigue syndrome. Free Radic Biol Med 29: 1252-1259, 2000

35. Pastore S, Mariani V, Lulli D, Gubinelli E, Raskovic D, Mariani S, Stancato A, de Luca C, Pecorelli A, Valacchi G, et al: Glutathione peroxidase activity in the blood cells of psoriatic patients correlates with their responsiveness to Efalizumab. Free Radic Res 45: 585-599, 2011.

36. Marantos C, Mukaro V, Ferrante J, Hii C and Ferrante A: Inhibition of the lipopolysaccharide-induced stimulation of the members of the MAPK family in human monocytes/macrophages by 4-hydroxynonenal, a product of oxidized omega- 6 fatty acids. Am J Pathol 173: 1057-1066, 2008.

37. Giusti B, Vestrini A, Poggi C, Magi A, Pasquini E, Abbate R and Dani C: Genetic polymorphisms of antioxidant enzymes as risk factors for oxidative stress-associated complications in preterm infants. Free Radic Res 46: 1130-1139, 2012.

38. Palmirotta R, Barbanti P, De Marchis ML, Egeo G, Aurilia C, Fofi L, Ialongo C, Valente MG, Ferroni P, Della-Morte D, et al: Is SOD2 Ala16Val polymorphism associated with migraine with aura phenotype? Antioxid Redox Signal 22: 275-279, 2015.

39. Hernando B, Gil-Barrachina M,Tomás-Bort E, Martinez-Navarro I, Collado-Boira $\mathrm{E}$ and Hernando $\mathrm{C}$ : The effect of long-term ultra-endurance exercise and SOD2 genotype on telomere shortening with age. J Appl Physiol (1985) 129: 873-879, 2020. 
40. Synowiec E, Wigner P, Cichon N, Watala C, Czarny P Saluk-Bijak J, Miller E, Sliwinski T, Zielinska-Nowak E and Bijak M: Single-nucleotide polymorphisms in oxidative stress-related genes and the risk of a stroke in a Polish population - a preliminary study. Brain Sci 11: 391, 2021.

41. Atanasovska Velkovska M, Goričar K, Blagus T, Dolžan V and Cvenkel B: Association of genetic polymorphisms in oxidative stress and inflammation pathways with glaucoma risk and phenotype. J Clin Med 10: 1148, 2021.

42. Crawford A, Fassett RG, Geraghty DP, Kunde DA, Ball MJ, Robertson IK and Coombes JS: Relationships between single nucleotide polymorphisms of antioxidant enzymes and disease. Gene 501: 89-103, 2012.

43. Kang SW: Superoxide dismutase 2 gene and cancer risk: Evidence from an updated meta-analysis. Int J Clin Exp Med 8: 14647-14655, 2015.

44. Azzolin VF, Barbisan F, Teixeira CF, Pillar D, Mastella MH, Duarte T, Turra BO, Ribeiro EE, Duarte MMFM and da Cruz IBM: The Val16Ala-SOD2 polymorphism affects cyto-genotoxicity of pyridostigmine bromide on human peripheral blood mononuclear cells. Toxicol In Vitro 60: 237-244, 2019.
45. Dornelles EB, Goncalves BD, Schott KL, Barbisan F, Unfer TC, Glanzner WG, Machado AK, Cadona FC, Azzolin VF, Montano MA, et al: Cytotoxic effects of moderate static magnetic field exposure on human periphery blood mononuclear cells are influenced by Val16Ala-MnSOD gene polymorphism. Environ Sci Pollut Res Int 24: 5078-5088, 2017.

46. Belpomme D, Campagnac C and Irigaray P: Reliable disease biomarkers characterizing and identifying electrohypersensitivity and multiple chemical sensitivity as two etiopathogenic aspects of a unique pathological disorder. Rev Environ Health 30: 251-271, 2015.

47. Irigaray P, Caccamo D and Belpomme D: Oxidative stress in electrohypersensitivity self reporting patients: Results of a prospective in vivo investigation with comprehensive molecular analysis. Int J Mol Med 42: 1885-1898, 2018.

48. Rossi S and Pitidis A: Multiple Chemical Sensitivity: Review of the State of the Art in Epidemiology, Diagnosis, and Future Perspectives. J Occup Environ Med 60: 138-146, 2018.

(i) This work is licensed under a Creative Commons

cc) Attribution-NonCommercial-NoDerivatives 4.0 International (CC BY-NC-ND 4.0) License. 